\title{
An Anatomical Study of Triangle of Brocq \& Mouchet in Human Cadaveric Heart \& Its Clinical Relevance
}

\author{
Bharathi $\mathrm{D}^{1}$., Sathyamurthy B. ${ }^{2}$ \\ ${ }^{I}$ (Postgraduate, Department of Anatomy, Rajarajeswari Medical College \& Hospital, Bangalore-560074, Rajiv \\ Gandhi University of Health Sciences, India) \\ ${ }^{2}$ (Professor \& HOD, Department of Anatomy, Rajarajeswari Medical College \& Hospital, Bangalore-560074, \\ Rajiv Gandhi University of Health Sciences, India)
}

\begin{abstract}
Arteriovenous triangle of Brocq \& Mouchet is formed by Great cardiac vein (GCV), Circumflex artery $(C A)$ and Anterior Interventricular artery (AIA). The latter two are branches of left coronary artery which lies on left side of anterior surface of the heart.

According to the pattern of disposition of vessels forming the triangle, the triangle is classified as closed, completely opened, inferiorly opened and superiorly opened. The triangle may also be absent.

Thirty human cadaveric hearts were studied by dissection method in the Department of Anatomy, RRMCH and $B M C \& R I$ in Bangalore. The triangle of Brocq \& Mouchet was identified and photographed.

During the study, the triangle of Brocq and Mouchet was present in twenty-six hearts (86.7\%) and was absent in four (13.3\%). In our study the most common was Closed type as seen in 15(50\%) and least common was Superiorly opened seen in 3(10\%) specimens.

There are several strategies in interventional cardiology tailored to the patients with Ischaemic heart disease. The anatomical knowledge of relations of these vessels provides a basis for newer cardiac interventional surgeries.
\end{abstract}

Keywords: Triangle of Brocq \& Mouchet, Great cardiac vein, Circumflex artery, Anterior Interventricular artery, Cardiac interventional surgeries.

\section{Introduction}

The left coronary artery divides into anterior interventricular artery which descends to the left in interventricular groove crossed by bridges of myocardial tissue, and by the great cardiac vein and its tributaries. The circumflex artery curves left in the atrioventricular groove, continuing round the left cardiac border and ending left of crux in most of the hearts, but sometimes continuing as posterior interventricular artery. The great cardiac vein begins at cardiac apex, ascends in the interventricular groove to the atrioventricular groove, passing to the left and posteriorly to enter the coronary sinus at its origin [1].

The Brocq and Mouchet's arteriovenous triangle is a well known topographic region that is composed by the intersection of the great cardiac vein, the circumflex artery and the anterior interventricular branches of the left coronary artery as shown in Fig. 1. These structures are located between conus arteriosus and the left auricle, on the anterior surface of the heart [2]. 


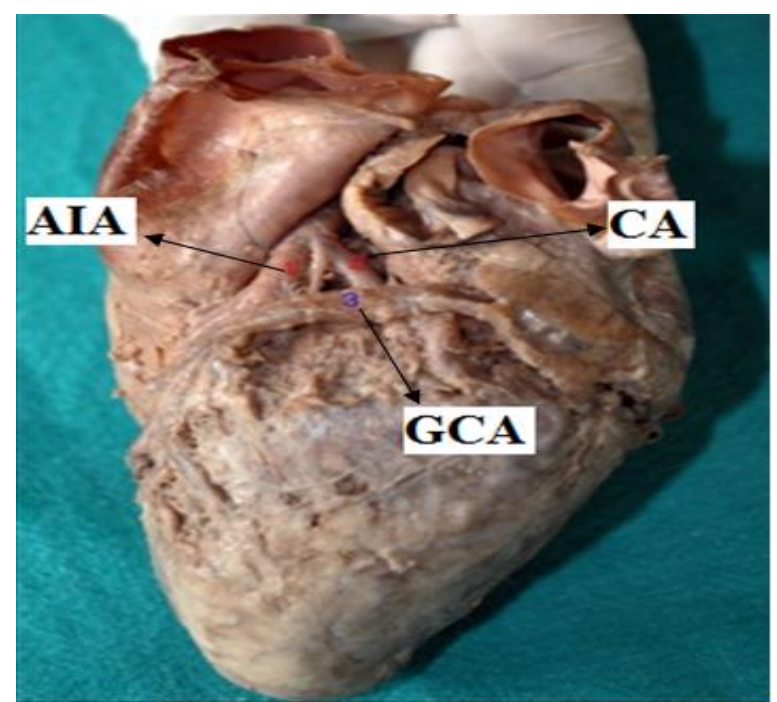

Fig. 1 - Closed Triangle seen in the heart specimen

1. AIA: Anterior Interventricular Artery, 2. CA: Circumflex Artery, 3. GCA: Great Cardiac Vein

The triangle was usually traversed by diagonal branches of the left anterior descending artery, which either passed superficial or deep to the vein. The latter part of the course of the great cardiac vein, as it left the triangle, lay in close relationship to the left circumflex artery: it passed from below upwards and to the right, then superficial to the artery. Thus the vein had a triple relation to the artery; being proximally inferior, then superficial, and finally superior to the artery [3].

On reaching the area of the coronary sulcus, the great cardiac vein crosses the anterior interventricular branch and the circumflex branch of the left coronary artery forming triangle of Brocq and Mouchet in which the vein is mainly superficial $(61 \%)$ [4].

The arteriovenous connections in the human heart should, moreover, not be underestimated, especially in the light of research strongly affirming the importance of the diagnosis and therapy of Ischemic myocardial lesions [5].

Routine radiographic protocols do not allow simultaneous study of coronary arterial and venous systems and hence their relationship [6].

The aim of the study was to know the relationship of the Great cardiac vein and the two branches of left coronary artery namely Anterior Interventricular \& Circumflex artery. It also emphasizes its potential value for new therapeutic approaches in invasive cardiology.

\section{Materials \& Methods}

The present study was carried out in the Department of Anatomy at Rajarajeswari Medical College \& Hospital and Bangalore Medical College \& Research Institute, Bangalore, Karnataka, India. Thirty human cadaveric hearts of both sexes fixed in $10 \%$ formalin were dissected to observe the triangle of Brocq and Mouchet. The vessels were traced and cleared. The various types of triangle depending on the disposition of blood vessels forming it were observed and photographed. The data obtained was analyzed and compared with that of previous studies.

\section{Results}

Out of thirty human cadaveric hearts studied, the triangle of Brocq and Mouchet was observed in 26 hearts $(86.7 \%)$ and was absent in $4(13.3 \%)$. In all specimens where triangle was present, Diagonal artery crossed it \& in 10 specimens there were minor left ventricular branches crossing it.

Regarding classification of triangle, the pattern of distribution in our study is as follows:

a. Closed- 15 specimens $(50 \%)$

b. Completely opened- $2(6.7 \%)$

c. Inferiorly opened- $6(20 \%)$

d. Superiorly opened- $3(10 \%)$

e. Absent - $4(13.3 \%)$ 

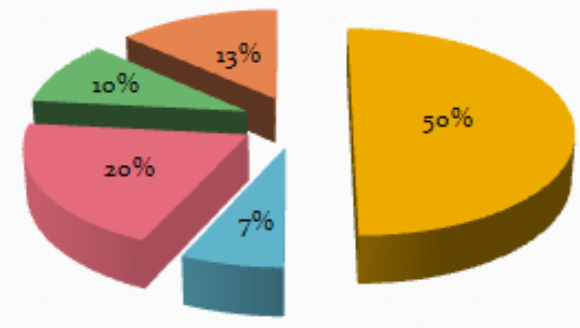

a Closed

a Completely opened

a Inferiorly opened

- Superiorly opened

absent

\section{The pie chart showing the frequency of occurrence of type of triangle}

\section{Discussion}

The triangle of Brocq \& Mouchet is subdivided following the recommendations of previous studies (Pejkovic and Bogdanovich, [4]) into-

a. Closed: when the GCV crosses both the CA and the AIA as shown in Fig.1;

b. Completely opened: the GCV does not cross any vessel and is located inferiorly to CA as shown in Fig. 2;

c. Superiorly opened: the GCV crosses the AIA (anteriorly or posteriorly) during its course at interventricular groove, but lies inferiorly to CA without crossing this vessel;

d. Inferiorly opened: the GCV is located on the left of AIA and doesn't cross this vessel at the interventricular groove. After leaving the interventricular groove and turning left, the vein crosses the CA, passing anteriorly or posteriorly to this vessel as shown in Fig. 3;

e. Absence of the triangle: when the GCV is on the left of AIA, ascends and then turns left, where it accompanies the CA as shown in Fig. 4.

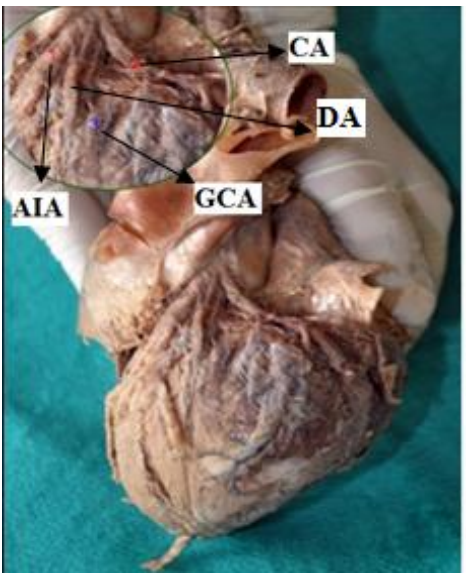

Fig.2 - Completely Opened Triangle (Inset shows the Diagonal Artery-DA)

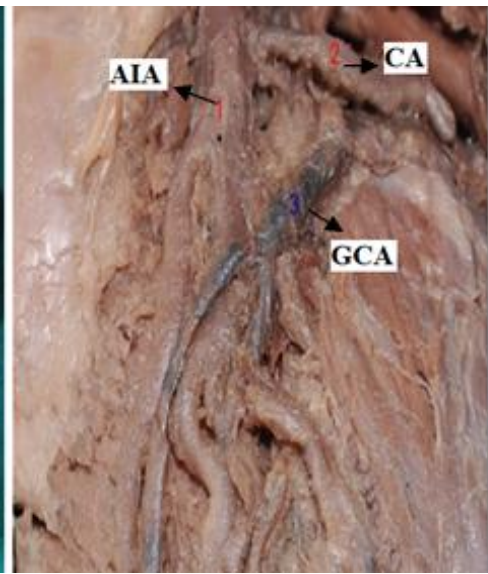

Fig.3 - Inferiorly Opened Triangle

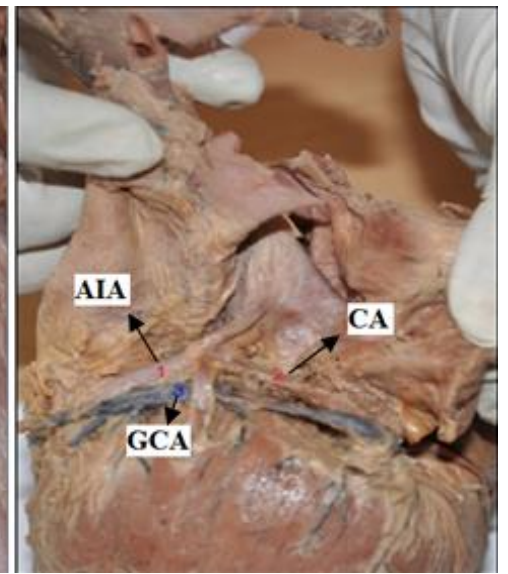

Fig.4 - Triangle is Absent

1. AIA: Anterior Interventricular Artery; 2. CA: Circumflex Artery; 3. GCA: Great Cardiac Vein

According to Andrade study [2], the triangle was found in $86.9 \%$ \& in remaining $11.1 \%$ it was absent. According to Pejkovic \& Bogdanovic study [4], who reported an occurrence of triangle in $98 \%$ of examined specimens, the GCV as it crosses the AIVA \& CA, the vein lies superficial in $61 \%$ of cases \& when the position of GCV is deeper than the arteries, it can result in pressure. According to Ortale study [7], the most common triangle was inferiorly opened

Commonly, the great cardiac vein varies with respect to presence, location and the superficial or deep relationship of single crossings of the anterior interventricular and circumflex arteries. The relations of GCV to CA and AIA, regarding to the type of crossing between vessels had been extensively studied, and the vein may cross those vessels anteriorly, posteriorly or never cross neither [8]. 
TABLE 1- Comparing the frequency of types of Brocq \& Mouchet triangle in various studies

\begin{tabular}{|l|l|l|l|}
\hline $\begin{array}{l}\text { Classification of the } \\
\text { Triangle }\end{array}$ & $\begin{array}{l}\text { Our Study } \\
\mathbf{\%}\end{array}$ & $\begin{array}{l}\text { Andrade, } \\
\text { Ribiero, } \\
\text { Babinski } \\
(\mathbf{2 0 1 0}) \%\end{array}$ & $\begin{array}{l}\text { Ortale, } \\
\text { Gabriel. } \\
\text { Lost } \\
(\mathbf{2 0 0 1}) \%\end{array}$ \\
\hline Closed & 50 & 22 & 18 \\
\hline Completely Opened & 6.6 & 22 & 15 \\
\hline Inferiorly Opened & 20 & 44 & 64 \\
\hline Superiorly Opened & 10 & 11 & 3 \\
\hline
\end{tabular}

There is global burden of cardiovascular disease with changing lifestyle \& stress. Among the myriad of options available like anti-ischaemic therapy and risk factor reduction, revascularization plays a vital role in Isheaemic heart disease.

Although revascularization procedures by percutaneous intervention or bypass surgery are largely used in the treatment of ischemic heart disease, there remains a relatively large number of patients with refractory angina. These "no options" patients frequently have diffuse coronary disease without a discrete target for angioplasty or surgical bypass [9].

Recently, numerous percutaneous interventional treatments and diagnostic tools have been developed to diagnose the vulnerable plaque and to treat the large number of patients with myocardial ischemia.

Catheter based coronary bypass which includes Percutaneous in situ coronary venous arterializations (PICVA), in which coronary artery is connected to adjacent vein at one site upstream from the lesion, directing oxygenated blood flow into the vein. The oxygenated blood then travels through the venous system in reverse direction to perfuse the heart.

In Percutaneous in-situ coronary artery bypass (PICAB), two channels are created between coronary artery and adjacent vein, one upstream and other downstream the lesion. Blood enters upstream channel, flows through isolated vein to bypass the lesion and then reenters the healthy segment of artery through downstream channel [10]

\section{Conclusion}

This anatomic study highlights the topography of arteriovenous triangle of Brocq and Mouchet and its application for above mentioned cardiac interventional surgeries. Hence the proximity of vein to artery is crucial for an effective surgery. Further anatomical studies are required to enhance the knowledge \& skills for development of advanced cardiac interventional procedures.

\section{Acknowledgements}

I express my sincere thanks to Prof Dr. Ramachandrappa G.K, Prof Dr. Balakrishna and Prof Dr. Komala B, Dept. of Anatomy, RRMCH for their valuable suggestion \& guidance which enabled me to do the study with ease. I am thankful to our institutional photographer Mr. Sathyan M.for taking high quality photographs. I wish to express my immense gratitude to my husband Mr. Nagaraj K.S for not just providing invaluable support; but also involved in typing \& editing this study.

\section{References}

[1] Standring S. Gray's Anatomy: The Anatomical Basis of Clinical Practice $40^{\text {th }}$ edition. (Edinburgh: Elsevier/ Churchill Livingstone. 2008).

[2] Andrade FM, Ribiero DC, Babinski MA, Cisne R, Goes ML .Triangle of Brocq \& Mouchet in Brazilian cadavers and clinical implications. J Morphol Sci, 27 (3-4), 2010, 127-129.

[3] El- Maasarany S, Colin GF, Firth A, Sheppard M, Michael Y. Henein. The coronary sinus conduit function: Anatomical study (relationship to adjacent structures) Europace, 7, 2005, 475-481.

[4] Pejkovic B, Bogdanovic D. The great cardiac vein. Surg radiol anat. 1992; 14(1):23-8.

[5] Kaczmarek M, Czerwinski F. Assessment of the course of the great cardiac vein in a selected number of patients. Folia Morphol, 666(3), 2007,190-193

[6] Schumacher B, Tebbenjohanns J, Pfeiffer D, Omrah H, Jung W, Luderitz B. Prospective study of retrograde coronary venography in patients with postero-septal and left sided accessory atrioventricular pathways. Am Heart J, 130, 2005,1031-9.

[7] Ortale JR, Gabriel EA, Lost C, Marquez C. The anatomy of coronary sinus and its tributaries. Surg Radio Anat,23(1), 2001,15-21.

[8] Bales GS. Great cardiac vein variations. Clin Anat, 17(5), 2004, 436-43.

[9] Siminiak T, Lipiecki J. Trans-coronary-venous interventions. Circ Cardiovasc Intervent, 1, 2008, 134-142.

[10] Aoki J, Rodriguez- Granillo GA, Serruys PW. Emergent strategies in interventional cardiology. Rev Esp Cardio, 58, 2005, 962-73. 